\title{
A Comparison of Some Taylor and Chebyshev Series
}

\author{
By R. E. Scraton
}

\begin{abstract}
A function is approximated in the interval $-1 \leq x \leq 1$ by (i) a Taylor series in $x$; (ii) a Taylor series in $y=(x+\lambda) /(1+\lambda x)$; (iii) a Chebyshev series in $x$; and (iv) a Chebyshev series in $z=(x+\mu) /(1+\mu x)$. The convergence of all four series is discussed, and a method is given for finding the values of $\lambda$ and $\mu$ which optimize convergence. Methods are also given for transforming one of the above series into another, some of which provide effective methods for acceleration of convergence. The application of the theory to even and odd functions is also discussed.
\end{abstract}

1. Introduction. In this paper we consider the use of the four series given below for approximating a function $f$ in the interval $-1 \leq x \leq 1$ :

$$
\begin{aligned}
& \text { (i) } f(x)=a_{0}+a_{1} x+a_{2} x^{2}+a_{3} x^{3}+\cdots ; \\
& \text { (ii) } f(x)=b_{0}+b_{1} y+b_{2} y^{2}+b_{3} y^{3}+\cdots,
\end{aligned}
$$

where

$$
y=\frac{x+\lambda}{1+\lambda x}, \quad|\lambda|<1, \lambda \in R
$$

$$
\text { (iii) } f(x)=c_{0}+c_{1} T_{1}(x)+c_{2} T_{2}(x)+c_{3} T_{3}(x)+\cdots,
$$

where $T_{r}(x)=\cos \left(r \cos ^{-1} x\right)$ is the Chebyshev polynomial; and

$$
\text { (iv) } f(x)=d_{0}+d_{1} T_{1}(z)+d_{2} T_{2}(z)+d_{3} T_{3}(z)+\cdots,
$$

where

$$
z=\frac{x+\mu}{1+\mu x}, \quad|\mu|<1, \mu \in R
$$

Note that, contrary to normal practice, the factor $\frac{1}{2}$ has not been included in the first terms of the Chebyshev series.

We shall treat $x$ as a complex variable. The region over which we wish to approximate $f(x)$, that is the segment of the real axis for which $-1 \leq x \leq 1$, will be denoted by $L$. We assume that $f(x)$ is real for $x \in L$, so that the coefficients $a_{r}, b_{r}, c_{r}, d_{r}$ are all real. The singularities of $f$, which will be denoted by $\alpha_{1}, \alpha_{2}, \alpha_{3}, \ldots$, are thus assumed to be placed symmetrically about the real axis.

2. Convergence of the Series. From elementary theory, we know that the series (i) converges in the region

$$
|x|<\rho_{1}
$$

Received May 27, 1986; revised December 15, 1986.

1980 Mathematics Subject Classification (1985 Revision). Primary 65B10; Secondary 41A50. 
where

$$
\rho_{1}=\min _{j}\left\{\left|\alpha_{j}\right|\right\}
$$

Similarly, series (ii) converges in the region

$$
\left|\frac{x+\lambda}{1+\lambda x}\right|<\rho_{2}(\lambda)
$$

where

$$
\rho_{2}(\lambda)=\min _{j}\left\{\left|\frac{\alpha_{j}+\lambda}{1+\lambda \alpha_{j}}\right|\right\} .
$$

Both series may also converge on the boundaries of the regions given, but we shall ignore such critical convergence in this paper. It is easily seen that neither series can converge in the entire region $L$ unless all the $\alpha_{j}$ lie outside the unit circle. Series (ii) has some resemblance to the Euler transformation; the method for optimizing its convergence given in the next section may be compared with the method for optimizing the Euler transformation, given previously by the present author [1].

The value of $\rho_{2}(\lambda)$ may be taken as a measure of the rate of convergence of series (ii) over the region $L$; the larger the value of this parameter, the more rapid the convergence. Thus the optimum value of $\lambda$ will be taken as the value which maximizes $\rho_{2}(\lambda)$. Unless this optimum value is zero, we obtain an improvement in convergence by using series (ii) rather than series (i).

The region of convergence of the Chebyshev series (iii) is the interior of an ellipse with foci at \pm 1 ; more precisely, it is the largest such ellipse for which all the $\alpha_{j}$ lie outside or on the ellipse. We can thus guarantee the convergence of series (iii) throughout $L$ provided that none of the $\alpha_{j}$ lie on $L$. In order to define the region of convergence algebraically, we introduce a function $k$ given by

$$
k(x)=x \pm \sqrt{\left(x^{2}-1\right)},
$$

where the sign is chosen so that $|k(x)|>1$ if $x \notin L$ and $\operatorname{Im}\{k(x)\} \geq 0$ if $x \in L$. The region of convergence of series (iii) can then be written as

$$
|k(x)|<\rho_{3}
$$

where

$$
\rho_{3}=\min _{j}\left\{\left|k\left(\alpha_{j}\right)\right|\right\} .
$$

The series (iv) has been discussed previously by the present author [2], and subsequently by Locher [3]. Its region of convergence is given by

$$
\left|k\left(\frac{x+\mu}{1+\mu x}\right)\right|<\rho_{4}(\mu),
$$

where

$$
\rho_{4}(\mu)=\min _{j}\left\{\left|k\left(\frac{\alpha_{j}+\mu}{1+\mu \alpha_{j}}\right)\right|\right\} .
$$

We may use $\rho_{4}(\mu)$ as a measure of the rate of convergence of series (iv). The optimum value of $\mu$ is the value which maximizes $\rho_{4}(\mu)$, and if this value differs from zero the optimal form of series (iv) converges more rapidly than series (iii). 
3. The Optimum Values of $\lambda$ and $\mu$. The determination of the value of $\lambda$ which maximizes $\rho_{2}(\lambda)$ is not a trivial problem. The geometric approach given below does not appear to be well known.

We transform the $x$-plane into the $u$-plane by means of the conformal transformation

$$
u=\frac{x-1}{x+1}, \quad x=\frac{1+u}{1-u}
$$

This transforms the exterior of the unit circle in the $x$-plane into the half plane $\operatorname{Re}\{u\}>0$. On the assumption that the $\alpha_{j}$ lie outside the unit circle, their images $\sigma_{j}$ defined by

$$
\sigma_{j}=\frac{\alpha_{j}-1}{\alpha_{j}+1}
$$

must lie in the right-hand half of the $u$-plane. The region of convergence (9) of series (ii) maps into the region

$$
\left|\frac{l+u}{l-u}\right|<\rho_{2}(\lambda)
$$

where

$$
l=\frac{1+\lambda}{1-\lambda}
$$

The region (18) is the exterior of a circle of the coaxial system with limiting points at $\pm l$. This circle is the smallest circle of the coaxial system for which all the $\sigma_{j}$ lie inside or on the circle. Suppose the tangents from the origin $O$ to this circle are $O T, O T^{\prime}$; then we can easily see that

$$
O T=l
$$

and

$$
\rho_{2}(\lambda)=\cot \left[\frac{1}{4} \widehat{T O T}^{\prime}\right]
$$

These results are true for any value of $\lambda$; we want to choose $\lambda$ to maximize $\rho_{2}(\lambda)$, or equivalently to minimize $\widehat{T O T}^{\prime}$. Thus we have to choose a circle so that all the $\sigma_{j}$ lie inside or on the circle, and so that this circle subtends as small an angle as possible at the origin. If $l$ is the length of the tangent from the origin to this circle, then the optimum value of $\lambda$ is given by

$$
\lambda=\frac{l-1}{l+1} .
$$

The optimum value of $\mu$ can be obtained in a manner (briefly indicated in [2]) which closely parallels the above work. We use the conformal transformation

$$
v^{2}=\frac{x-1}{x+1}, \quad x=\frac{1+v^{2}}{1-v^{2}} .
$$

This transforms the whole of the $x$-plane, except for the line segment $L$, into the right-hand half of the $v$-plane, $\operatorname{Re}\{v\}>0$. The singularities $\alpha_{j}$ (which are assumed not to lie on $L$ ) map into $\tau_{j}$, where

$$
\tau_{j}^{2}=\frac{\alpha_{j}-1}{\alpha_{j}+1}, \quad \operatorname{Re}\left\{\tau_{j}\right\}>0
$$


The region (14) in which series (iv) converges maps into

$$
\left|\frac{m+v}{m-v}\right|<\rho_{4}(\mu)
$$

where

$$
m^{2}=\frac{1+\mu}{1-\mu}, \quad m>0 .
$$

We can now proceed exactly as before. We choose a circle such that all the $\tau_{j}$ lie inside or on the circle, and so that this circle subtends as small an angle as possible at the origin. If $m$ is the length of the tangent from the origin to this circle, then the optimum value of $\mu$ is given by

$$
\mu=\frac{m^{2}-1}{m^{2}+1}
$$

As an example, consider $f(x)=\ln (p+q x)$, where $p>|q|>0$. This is given as a numerical example in [2] with $p=5.5$ and $q=4.5$. Formulae for the coefficients $c_{r}, d_{r}$ in this case are given by Locher [3], but unfortunately are incorrect; correct formulae are given below. The singularities of this function are at $-p / q, \infty$, and the treatment above shows that the optimum values of $\lambda$ and $\mu$ are both equal to $\left[p-\sqrt{\left(p^{2}-q^{2}\right)}\right] / q=w$, say. The coefficients in series (i)-(iv) are then given by

$$
\begin{array}{rlrl}
a_{0} & =\ln p ; & a_{r} & =\frac{1}{r}(-1)^{r-1}\left(\frac{q}{p}\right)^{r}, \quad r \geq 1 ; \\
b_{0} & =\frac{1}{2} \ln \left(p^{2}-q^{2}\right) ; & b_{r} & =\frac{2}{r} w^{r}, \quad r \text { odd } ; \quad b_{r}=0, \quad r \text { even, } r \geq 2 ; \\
c_{0} & =\ln \frac{q}{2 w} ; & c_{r} & =\frac{2}{r}(-1)^{r-1} w^{r}, \quad r \geq 1 ; \\
d_{0} & =\frac{1}{2} \ln \left(p^{2}-q^{2}\right) ; & d_{r} & =\frac{4}{r}\left[\frac{1-\sqrt{\left(1-w^{2}\right)}}{w}\right]^{r}, \quad r \text { odd } ; \\
d_{r}=0, \quad r \text { even, } r \geq 2 .
\end{array}
$$

In the case $p=5.5, q=4.5$, we can get 12 decimal place accuracy by going as far as $a_{117}$ in series (i), $b_{37}$ in series (ii), $c_{38}$ in series (iii), and $d_{19}$ in series (iv).

4. Transformation of One Series into Another. Series (i) is the usual Taylor series and can be obtained by elementary means. The other three series can be obtained quite easily if series (i) is known. In fact, there are a number of methods for transforming one of the four series into another, and we summarize these below. Insofar as series (ii) and (iv) are generally more rapidly convergent than series (i) and (iii), some of these transformations may be regarded as methods for accelerating the convergence of series. They may also be regarded as methods for analytic continuation, since in many cases the transformed series converges in a larger region than the original series.

Locher [3] has shown that series (iii) can be transformed into series (iv) by a transformation which may be written

$$
d_{r}=\sum_{s=0}^{\infty} p_{r, s}\left(\frac{1-\sqrt{\left(1-\mu^{2}\right)}}{\mu}\right) c_{s}
$$


where the functions $p_{r, s}$ are given by

$$
\begin{gathered}
p_{0, s}(\xi)=(-\xi)^{s}, \quad s \geq 0 \\
p_{r, 0}(\xi)=0, \quad r \geq 1, \\
p_{r, s}(\xi)=\xi p_{r-1, s}(\xi)-\xi p_{r, s-1}(\xi)+p_{r-1, s-1}(\xi), \quad r \geq 1, s \geq 1 .
\end{gathered}
$$

By methods similar to those used by Locher, we can transform series (i) into series (ii), series (ii) into series (i), and series (iv) into series (iii), thus:

$$
\begin{gathered}
b_{r}=\sum_{s=0}^{\infty} p_{r, s}(\lambda) a_{s}, \\
a_{r}=\sum_{s=0}^{\infty} p_{r, s}(-\lambda) b_{s}, \\
c_{r}=\sum_{s=0}^{\infty} p_{r, s}\left(-\frac{1-\sqrt{\left(1-\mu^{2}\right)}}{\mu}\right) d_{s} .
\end{gathered}
$$

It is easily shown that series (32) and (33) converge if $f$ has no singularities inside the unit circle, whilst (28) and (34) converge if there are no singularities on $L$.

By making use of the identity

$$
(2 x)^{s}=\sum_{r=0}^{s}\left(\begin{array}{l}
s \\
r
\end{array}\right) T_{|2 r-s|}(x)
$$

we can show that

$$
c_{r}=\sum_{s=0}^{\infty} q_{r, s} a_{r+2 s}
$$

where

$$
q_{0, s}=2^{-2 s}\left(\begin{array}{c}
2 s \\
s
\end{array}\right), \quad q_{r, s}=2^{-r-2 s+1}\left(\begin{array}{c}
r+2 s \\
s
\end{array}\right), \quad r \geq 1 .
$$

This enables us to transform series (i) into series (iii). Provided that we choose $\lambda=\mu$, we can also take

$$
d_{r}=\sum_{s=0}^{\infty} q_{r, s} b_{r+2 s}
$$

thereby transforming series (ii) into series (iv). Series (36) is convergent if $f$ has no singularities inside or on the unit circle. If series (i) converges slowly near $|x|=1$, then series (36) also converges slowly; in this case, it may be better to obtain series (iii) by using Eqs. (32), (38), (34) in sequence, rather than by direct use of Eq. (36).

5. Even and Odd Functions. If the singularities of $f$ are symmetrically placed about the imaginary axis, the optimum values of $\lambda$ and $\mu$ are zero, and no improvement in convergence is obtained by using series (ii) and (iv) instead of series (i) and (iii). This situation most frequently arises when $f$ is an even or an odd function; in this case we can make use of the above results by proceeding as follows. 
Let $g$ be an even or odd function with Taylor series

$$
g(t)=h_{0}+h_{1} t^{2}+h_{2} t^{4}+h_{3} t^{6}+\cdots
$$

or

$$
g(t)=h_{0} t+h_{1} t^{3}+h_{2} t^{5}+h_{3} t^{7}+\cdots
$$

and Chebyshev series

$$
g(t)=k_{0}+k_{1} T_{2}(t)+k_{2} T_{4}(t)+k_{3} T_{6}(t)+\cdots
$$

or

$$
g(t)=k_{0} T_{1}(t)+k_{1} T_{3}(t)+k_{2} T_{5}(t)+k_{3} T_{7}(t)+\cdots
$$

Define

$$
f(x)=h_{0}+h_{1}\left(\frac{1+x}{2}\right)+h_{2}\left(\frac{1+x}{2}\right)^{2}+h_{3}\left(\frac{1+x}{2}\right)^{3}+\cdots,
$$

so that $g(t)=f\left(2 t^{2}-1\right)$ if $g$ is even, or $g(t)=t f\left(2 t^{2}-1\right)$ if $g$ is odd. We can then expand $f(x)$ by any of the four series (i)-(iv), and the resulting series can be used to evaluate $g(t)$ for $-1 \leq t \leq 1$.

In particular, series (ii) can be obtained by means of the formula

$$
b_{r}=\sum_{s=0}^{\infty} \pi_{r, s}(\lambda) h_{s}
$$

where

$$
\begin{gathered}
\pi_{0, s}(\lambda)=\left(\frac{1-\lambda}{2}\right)^{s}, \quad s \geq 0, \\
\pi_{r, 0}(\lambda)=0, \quad r \geq 1, \\
\pi_{r, s}(\lambda)=\lambda \pi_{r-1, s}(\lambda)+\frac{1}{2}(1-\lambda)\left[\pi_{r-1, s-1}(\lambda)+\pi_{r, s-1}(\lambda)\right], \\
r \geq 1, s \geq 1 .
\end{gathered}
$$

Series (i) can be computed directly using

$$
a_{r}=\sum_{s=0}^{\infty} \pi_{r, s}(0) h_{s}
$$

or from series (ii) using Eq. (33). Series (iii) and (iv) can then be obtained using Eqs. (36) and/or (38).

It should be noted that series (iii) is related to the original Chebyshev series (41) or (42). In fact $k_{r}=c_{r}$ if $g$ is even, whilst

$$
k_{0}=c_{0}+\frac{1}{2} c_{1} ; \quad k_{r}=\frac{1}{2}\left(c_{r}+c_{r+1}\right), \quad r \geq 1 ;
$$

if $g$ is odd. If series (39) or (40) is slowly convergent, the use of Eqs. (44), (38), (34) in sequence can be an efficient means of obtaining the Chebyshev series (41) or (42).

As a final example, consider $g(t)=\tan ^{-1} t$. For this function,

$$
h_{r}=\frac{(-1)^{r}}{2 r+1}, \quad k_{r}=\frac{2(-1)^{r}}{2 r+1}(\sqrt{2}-1)^{2 r+1} .
$$


In order to obtain 12 decimal place accuracy, we need to take $10^{12}$ terms of series (40), or continue as far as the term containing $T_{29}(t)$ in series (42). By using series (i)-(iv) obtained as above, we need to go only as far as the terms containing $a_{22}, b_{14}, c_{14}$ and $d_{10}$. The optimum values of $\lambda$ and $\mu$ are both equal to $(\sqrt{2}-1)^{2}$.

Another approach to even and odd functions is to use the procedure described in [2] to optimize the Chebyshev series over the interval $0 \leq t \leq 1$. Locher [3] gives the coefficients for the function $\tan ^{-1}$ obtained in this way, but again his results are in error: the correct formula is

$$
\tan ^{-1} t=\frac{\pi}{8}+\sum_{r=0}^{\infty} \frac{2(-1)^{r}[\sqrt{(4+2 \sqrt{2})}-\sqrt{2}-1]^{2 r+1}}{2 r+1} T_{2 r+1}\left(\frac{(\sqrt{2}+1) t-1}{(\sqrt{2}-1) t+1}\right)
$$

This last series gives 12 decimal accuracy with terms up to the one containing $T_{15}$. Since, for this particular function, the even terms in the series are zero, this is a useful series for $\tan ^{-1} t$.

Mathematics Department

University of the South Pacific

P. O. Box 1168

Suva, Fiji

1. R. E. Scraton, "A note on the summation of divergent power series," Proc. Cambridge Philos. Soc., v. 66, 1969, pp. $109-114$.

2. R. E. SCRATON, "A method for improving the convergence of Chebyshev series," Comput. J., v. 13, 1970, pp. 202-203.

3. F. LOCHER, "Accelerating the convergence of Chebyshev series," Computing, v. 15, 1975, pp. 235-246. 